\title{
The antimicrobial activity of ceftobiprole against Methicillin- resistant Staphylococcus aureus and multi-drug resistant Pseudomonas aeruginosa
}

\section{A large tertiary care university hospital experience in Riyadh, Saudi Arabia}

Lamees A. Altamimi, MBBS, Leen A. Altamimi, MBBS, Ali M. Somily, MD.

\begin{abstract}
الأهداف : تقييم فعالية السفتوبيبرول (ceftobiprole) ضد بكتينة بكتيريا

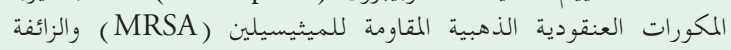

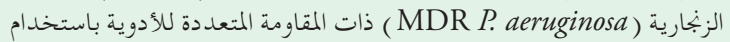
أنواع مختلفة من العينات و أنماط مختلفة من المقاومة ألمارمة المبعدة

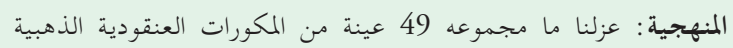

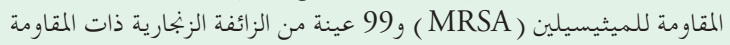

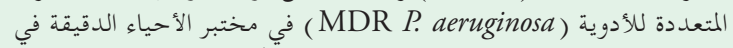

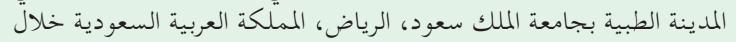

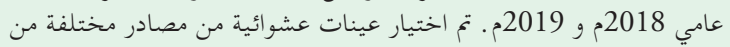

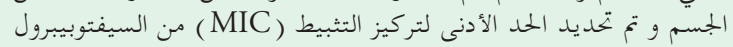

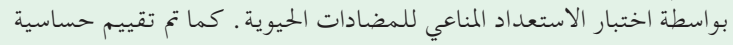

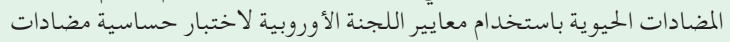
الميكروبات (EUCAST)

النتائج: وجدنا 100\% من عينات المكينات

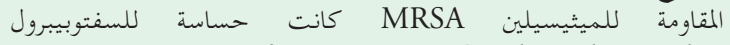
بينما 69.8\% من عينات الزائفة الزبارية (MIC 50/90: 1/1.5 mg/L)

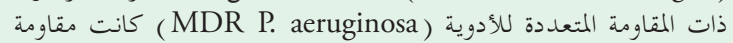

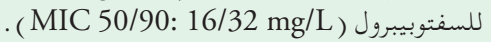

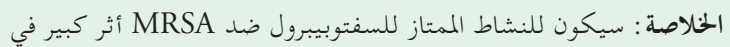

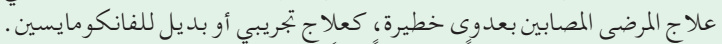

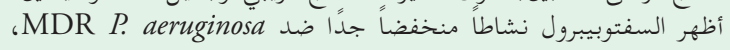

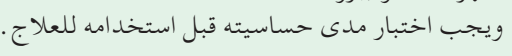

Objectives: To assess the antibacterial activity of ceftobiprole against Methicillin-resistant Staphylococcus aureus (MRSA) and Pseudomonas aeruginosa (P. aeruginosa) from various body specimen types and different patterns of resistance.

Methods: A retrospective cohort study with a total of 49 MRSA and $99 P$. aeruginosa isolated in the Microbiology Laboratory at King Saud University Medical City, Riyadh, Saudi Arabia, between 2018-2019, were used. Isolates were randomly selected from various specimen types. The minimum inhibition concentration (MIC) of ceftobiprole was determined by E-test. Breakpoints carried out by the European Committee on Antimicrobial Susceptibility Testing (EUCAST) were used to assess antibiotic susceptibility.

Results: Approximately $100 \%$ of the MRSA isolates were susceptible with MIC50/90 value of $1 / 1.5 \mathrm{mg} / \mathrm{L}$ while $69.8 \%$ of multi-drug resistant (MDR) $P$. aeruginosa isolates were resistant with MIC50/90 value of $16 / 32 \mathrm{mg} / \mathrm{L}$.

Conclusion: The excellent activity of ceftobiprole against MRSA would have major implications in management of the patients with serious infections, as an empirical treatment or alternative to vancomycin. Ceftobiprole has a very low activity against MDR P. aeruginosa, and its susceptibility should be tested prior to use for treatment.

Keywords: ceftobiprole, MRSA, MDR P. aeruginosa, tertiary care

Saudi Med J 2022; Vol. 43 (1): 31-36 doi: 10.15537/smj.2022.43.1.20210587

From the College of Medicine (Lamees Altamimi, Leen Altamimi), and from the Department of Pathology and Laboratory Medicine (Somily), King Saud University, King Saud University Medical City, Riyadh, Kingdom of Saudi Arabia.

Received 21st July 2021. Accepted 23rd November 2021.

Address correspondence and reprint request to: Dr. Ali M. Somily, Professor and Consultant Microbiologist, College of Medicine, King Saud University, King Saud University Medical City, Riyadh, Kingdom of Saudi Arabia.E-mail: ali.somily@gmail.com ORCID ID: https://orcid.org/0000-0002-9634-6107 
$\mathrm{T}$ he recent rise in Methicillin-resistant $S$. aureus (MRSA) and multi-drug resistant Pseudomonas aeruginosa (MDR P. aeruginosa) infections in hospitals and community settings is a serious issue worldwide. ${ }^{1,2}$ Due to the remarkable resistance to several commonly used antibiotics, eradication and treatment of bacterial infections has become a challenge. ${ }^{1,2}$ Many reports from Saudi Arabia showed a significant increase in MRSA and $P$. aeruginosa resistance during the previous decade. ${ }^{3}$

Only a few antibiotics have been approved for the treatment of MRSA and MDR P. aeruginosa. Vancomycin with individual dosing is an acceptable treatment option. ${ }^{4,5}$ Inadequate vancomycin doses and extended therapy, on the other hand, can result in an increase in the minimum inhibitory concentration (MIC), which can lead to therapeutic failure and toxicity. ${ }^{6}$ Linezolid and daptomycin have been used, but resistance was reported only after 5 years, which led to the need for new antibiotics. ${ }^{7}$ Cefazolin is an antibiotic that can be used to treat a variety of Gram-positive and Gram-negative infections, including MRSA and MDR $P$. aeruginosa, and has a broad spectrum of activity. ${ }^{8}$ Cefazolin was approved as a clinical and microbiological cure, but there was limited evidence of its usage in pediatric, pregnant or breastfeeding patients, and it is contraindicated in those with severe beta-lactams hypersensitivity. ${ }^{8}$

Ceftobiprole is a novel fifth-generation cephalosporin with a broad-spectrum antimicrobial activity against various bacteria such as MRSA and MDR P. aeruginosa. ${ }^{9-11}$ A total of 17 European countries and 8 non-European countries have authorized ceftobiprole for the treatment of community-acquired pneumonia (CAP) and hospital-acquired pneumonia (HAP), except ventilator-associated pneumonia, caused by Gram-positive and Gram-negative infections. ${ }^{12,13}$ Ceftobiprole is recommended as a parenteral therapy for the empirical treatment of hospitalized CAP patients who have risk factors for MRSA or P. aeruginosa. ${ }^{14}$ Moreover, it exhibits good activity against Staphylococcus aureus (S. aureus) isolated from HAP. ${ }^{15,16}$

Ceftobiprole has been proven to have a low possibility of causing antibiotic resistance. ${ }^{17,18}$ In addition, it has a high binding sensitivity for penicillin-binding protein $2 \mathrm{a}$, a special transpeptidase that is not inhibited with $\beta$-lactam antibiotics, which

Disclosure. Authors have no conflict of interests, and the work was not supported or funded by any drug company. increases its bactericidal efficacy against almost all MRSA strains. ${ }^{19}$

The objective of this study was to assess the antibacterial activity of ceftobiprole against MRSA and $P$. aeruginosa from various body specimen types and different patterns of resistance.

Methods. A retrospective cohort study with a total of 49 MRSA and 99 MDR $P$. aeruginosa isolated in the Microbiology Laboratory at King Saud University Medical City, Riyadh, Saudi Arabia, from January 2018 until December 2019 were included in the study.

Isolates were randomly selected from various specimen types, including body fluids, sputum, endotracheal secretions, blood, wound swabs, and urine. Isolates were refrigerated at $-80^{\circ} \mathrm{C}$ in skimmed milk, and each sample was rolled for at least 20 seconds on sheep blood agar (SBA) to guarantee that all sides of the specimen encountered the culture media. The SBA plates were incubated for 24 hours in an atmosphere containing $5 \% \mathrm{CO}_{2}$ at $35^{\circ} \mathrm{C}$. Definitive identification of methicillin-sensitive $S$. aureus and MRSA was carried out using automated identification and susceptibility testing. MicroScan Combo Panel (Vitek2 AST-N292 Microscan [Negative breakpoint combo 50]) was used for Pseudomonas. Acquired non-susceptibility of MDR organisms was defined as resistance to at least one agent from 3 or more antimicrobial categories. ${ }^{20}$

Susceptibility testing was carried out according to the Clinical and Laboratory Standards Institute (CLSI) guidelines. ${ }^{21}$ The E-test (BioMérieux, France) was used to determine the MIC of ceftobiprole on Mueller-Hinton agar (Saudi Prepared Media [SPM.co]). The European Committee on Antimicrobial Susceptibility Testing (EUCAST) breakpoints and M100-S28 were used for ceftobiprole susceptibility interpretations, $S$. aureus (susceptible: $\leq 2 \mathrm{~g} / \mathrm{ml}$; resistant: $>2 \mathrm{~g} / \mathrm{ml}$ ) and nonspecies-specific breakpoints (susceptible: $\leq 4 \mathrm{~g} / \mathrm{ml}$; resistant: $>4 \mathrm{~g} / \mathrm{ml}){ }^{22}$

Quality control criteria for ceftobiprole $5 \mu \mathrm{g}$ were carried out for $S$. aureus American Type Culture Collection ATCC 29213 and P. aeruginosa ATCC 27853 according to EUCAST SOP 9.0. ${ }^{21}$

Ethical approval was obtained from the Institutional Review Board of King Saud University, Riyadh, Saudi Arabia.

Statistical analysis. Minimum inhibitory concentration ranges (MIC50, MIC90) were presented by pathogen or pathogen group. There were no statistical analyses in this descriptive study.

Results. A total of 49 MRSA isolates were collected. Most isolates were from male patients 
between 20-39 years. Table 1 summarizes the pathogen distribution based on central laboratory identification and reflects the collection design. Isolates from the intensive care unit (ICU) accounted for $2.1 \%$ of the sample. Most of the samples were cultured from wounds.

Minimum inhibitory concentration and E-test results of ceftobiprole against MRSA are shown in Table 2. All 49 (100\%) tested isolates were susceptible (MIC: $\leq 2 \mathrm{mg} / \mathrm{L}$ ) with MIC50/90 value of $1 / 1.5 \mathrm{mg} / \mathrm{L}$.

A total of 99 MDR $P$. aeruginosa isolates were collected. The majority were from male patients aged 70-79 years. Table 3 shows the distribution of pathogens using central laboratory identification and reflects the collection design. A total of $15.2 \%$ of the isolates were from patients in the ICU. Most samples were cultured from respiratory sources.

Table 1 - Methicillin-resistant Staphylococcus aureus isolate and patient characteristics.

\begin{tabular}{lc}
\hline Characteristics & $\mathbf{n}(\%)$ \\
\hline Total number of isolates & 49 \\
Male gender & $33(67.3)$ \\
Age, years & \\
$0-4$ & $8(16.3)$ \\
$5-19$ & $6(12.3)$ \\
$20-39$ & $16(32.7)$ \\
$40-49$ & $6(12.3)$ \\
$50-59$ & $2(4.1)$ \\
$60-69$ & $7(14.3)$ \\
$70-79$ & $3(6.1)$ \\
$\geq 80$ & $1(2.0)$ \\
Source & \\
Respiratory & $7(14.3)$ \\
Blood & $5(10.2)$ \\
Urine & $2(4.1)$ \\
Wound & $27(55.1)$ \\
Body fluid & $2(4.1)$ \\
Tissue & $1(2.1)$ \\
Swab & $5(10.2)$ \\
Infection type & \\
ICU & $1(2.1)$ \\
\hline & \\
\hline
\end{tabular}

Table 2 - Distribution of ceftobiprole MIC by E-test of 49 isolates of MRSA.

\begin{tabular}{lccccc}
\hline $\begin{array}{l}\text { MIC } \\
(\mathrm{mg} / \mathrm{L})\end{array}$ & $\begin{array}{c}\text { No. of } \\
\text { isolates }\end{array}$ & Percentage & MIC50 & MIC90 & MIC50/90 \\
\hline 0.75 & 8 & 16.3 & & & \\
1 & 23 & 46.9 & 1 & 1.5 & 0.7 \\
1.5 & 15 & 30.6 & & & \\
2 & 3 & 6.1 & & \\
\hline \multicolumn{5}{c}{ MIC: minimum inhibitory concentration, No: number, } \\
\end{tabular}

Table 4 presents the E-test results and MIC of ceftobiprole against MDR P. aeruginosa. Of the 99 tested isolates, $30(30.2 \%)$ were susceptible (MIC: $\leq 4 \mu \mathrm{g} / \mathrm{mL}$ ), while 69 (69.8\%) were resistant (MIC: $\geq 4 \mu \mathrm{g} / \mathrm{mL}$ ) with MIC50/90 value of $16 / 32 \mathrm{mg} / \mathrm{L}$.

Table 5 shows the antimicrobial resistance patterns of MDR P. aeruginosa isolates. Nearly $50 \%$ of the isolates are

Table 3 - Multi-drug resistant $P$. aeruginosa isolate and patient's characteristics.

\begin{tabular}{lc}
\hline Characteristics & $\mathbf{n}(\%)$ \\
\hline Total number of isolates & 99 \\
Male gender & $54(54.5)$ \\
Age, years & \\
$0-4$ & $12(12.1)$ \\
$5-19$ & $7(7.1)$ \\
$20-39$ & $18(18.2)$ \\
$40-49$ & $2(2.0)$ \\
$50-59$ & $16(16.2)$ \\
$60-69$ & $13(13.1)$ \\
$70-79$ & $19(19.2)$ \\
$\geq 80$ & $12(12.1)$ \\
Source & \\
Respiratory & $42(42.4)$ \\
Blood & $0(0.0)$ \\
Urine & $24(24.2)$ \\
Wound & $22(22.2)$ \\
Body fluid & $7(7.1)$ \\
Tissue & $3(3.0)$ \\
Swab & $1(1.0)$ \\
Infection type & \\
ICU & $15(15.2)$ \\
\hline P. aeruginosa: Pseudomonas aeruginosa, ICU: intensive care unit \\
\end{tabular}

Table 4 - Distribution of ceftobiprole MIC by E-test of 99 isolates of MDR P. aeruginosa.

\begin{tabular}{cccccc}
\hline MIC $(\mathrm{mg} / \mathrm{L})$ & $\begin{array}{c}\text { No. of } \\
\text { isolates }\end{array}$ & Percentage & MIC50 & MIC90 MIC50/90 \\
\hline 0.047 & 1 & 1.01 & & & \\
0.094 & 1 & 1.01 & & & \\
1 & 3 & 3.03 & & & \\
1.5 & 2 & 2.02 & & & \\
2 & 2 & 2.02 & & & \\
3 & 10 & 10.10 & & & \\
4 & 11 & 11.11 & 16 & 32 & 0.5 \\
6 & 4 & 4.04 & & & \\
8 & 4 & 4.04 & & & \\
12 & 6 & 6.06 & & & \\
16 & 4 & 4.04 & & & \\
32 & 46 & 46.46 & & \\
$>32$ & 5 & 5.05 & & \\
\hline
\end{tabular}

MIC: minimum inhibitory concentration, MDR: multi-drug resistant, No: number, P. aeruginosa: Pseudomonas aeruginosa 
Table 5 - Antibiotic susceptibility patterns of tested MDR P. aeruginosa (N=99).

\begin{tabular}{lccc}
\hline Antibiotic suitability pattern & Total tested & \multicolumn{2}{c}{ MDR P. aeruginosa } \\
\hline & & Susceptible & Resistant \\
Ceftazidime & 81 & $56(56.6)$ & $25(25.6)$ \\
Piperacillin/tazobactam & 67 & $51(51.5)$ & $16(16.2)$ \\
Imipenem & 50 & $11(11.1)$ & $39(39.4)$ \\
Meropenem & 47 & $50(10.1)$ & $37(37.4)$ \\
Gentamicin & 80 & $50(50.5)$ & $30(30.3)$ \\
Tobramycin & 63 & $45(45.5)$ & $13(13.1)$ \\
Amikacin & 57 & $55(55.6)$ & $12(12.1)$ \\
Ciprofloxacin & 81 & $26(26.3)$ \\
\hline
\end{tabular}

MDR P. aeruginosa: Multi-drug resistant Pseudomonas aeruginosa

non-susceptible to ceftazidime, piperacillin/tazobactam, aminoglycoside, and ciprofloxacin, while only $10 \%$ are resistant to carbapenem.

American Type Culture Collection microbial reference strains were used to provide high quality control. Quality control criteria for ceftobiprole $5 \mu \mathrm{g}$ were carried out for $S$. aureus ATCC 29213 and P. aeruginosa ATCC 27853. The E-test was carried out to detect the MIC for both S. aureus 29213 and P. aeruginosa 27853. The results were $0.38 \mathrm{mg} / \mathrm{L}$ (susceptible) in $S$. aureus 29213 and $4 \mathrm{mg} / \mathrm{L}$ (susceptible) in P. aeruginosa 27853.

Discussion. Methicillin-resistant $S$. aureus infections are common medical problems that cause major consequences and have a higher morbidity and mortality rate than other bacterial infections. ${ }^{3}$ Clinical trials have led to the use of ceftobiprole for the empirical regimen and tailored therapy of CAP and HAP due to its activity against MRSA, favorable safety profile, and broad-spectrum activity. ${ }^{12,23}$

A recently published case series described a clinical trial treating MRSA bacteremia with ceftobiprole in combination with vancomycin. Results were promising, and clinical outcomes were excellent even when the patient had endocarditis and was immunocompromised. ${ }^{24}$ However, there is limited evidence for ceftobiprole use in Saudi Arabia. Also, there is a lack of studies that investigate the in vitro activity of ceftobiprole against MRSA and $P$. aeruginosa in Saudi Arabia or the Middle East.

In this study, $49(100 \%)$ of the MRSA isolates were susceptible to ceftobiprole with MIC50/90: 1/1.5mg/L. Our results are compatible with previous studies that reported susceptibility percentages. A study in Italy found only a $12 \%$ resistance rate to ceftobiprole $(12 / 102$ isolates; MIC: $\geq 4 \mathrm{mg} / \mathrm{L}) .^{25}$ A similar susceptibility rate was reported in Europe and Turkey
(98.3\% susceptible). ${ }^{26}$ Susceptibility of approximately 99.3\% was reported in the United States (US). ${ }^{10}$ Thus, ceftobiprole had a significant in vitro activity against MRSA.

Infection with MDR P. aeruginosa strains has become more common in recent years, posing a threat to public health. These strains cause serious infection, especially in hospital settings and in immuno-compromised patients. ${ }^{27}$ Ceftobiprole shows potent activity and great coverage of Gram-positive pathogens, such as MRSA. It has a range of action against Gram-negative bacilli such as MDR P. aeruginosa. ${ }^{10,27}$ However, there are no Saudi clinical trials to assess the effect of ceftobiprole against P. aeruginosa.

In our study, we found that only 30 (30.2\%) of MDR $P$. aeruginosa isolates were susceptible (MIC: $\leq 4 \mu \mathrm{g} / \mathrm{mL}$ ) while $69(69.8 \%)$ of the isolates were resistant with MIC50/90 value of 16/32mg/L. Previous studies have identified ceftobiprole's activity against MDR $P$. aeruginosa at a variety of susceptibility levels. A study in the Middle East, reported $62.4 \%$ susceptibility for ceftobiprole against $P$. aeruginosa. ${ }^{28}$ The potential of ceftobiprole against $P$. aeruginosa (MIC50/90 value of $2 />8 \mu \mathrm{g} / \mathrm{ml} ; 64.6 \%$ at MIC value of $\leq 4 \mu \mathrm{g} / \mathrm{ml}$ ) was reported in a European and Turkish study. ${ }^{26} \mathrm{~A}$ US study reported that approximately $72.7 \%$ of the $P$. aeruginosa isolates were susceptible to ceftobiprole. ${ }^{10}$ The notable discrepancy in the reported susceptibility percentages was likely multifactorial and might be due to the difference in sample sizes or sources.

A Turkish study compared the antimicrobial activity of fifth-generation cephalosporin, ceftaroline (both of which have recently received approval from the US Food and Drug Administration for therapeutic purposes), and ceftobiprole. The MIC50 and MIC90 values of all ceftaroline and ceftobiprole isolates were comparable to each other and defined as $0.50 \mu \mathrm{g} / \mathrm{ml}$ 
and $1 \mu \mathrm{g} / \mathrm{ml}$. For both antibiotics, the in vitro activities against MRSA indicated that they should be effective therapeutic options. ${ }^{29}$

Study limitations. The collected data was from a single center rather than multiple sites, and the sample size was small. The findings were not compared to the microdilution which is the gold standard way of measuring an antimicrobial agent's in vitro activity against a bacterial isolate. Nonetheless, this study provides useful preliminary data regarding the in vitro antibacterial activity of ceftobiprole in Saudi Arabia and the surrounding regions.

In conclusion, based on the E-test results, we found that ceftobiprole provides comprehensive coverage of MRSA and has limited activity against MDR $P$. aeruginosa. Although the clinical effectiveness of ceftobiprole for MRSA and MDR $P$. aeruginosa have been reported in some international studies, we recommend a larger multi-center clinical and laboratory study, together with local studies, to confirm ceftobiprole efficacy against MRSA and MDR P. aeruginosa strains.

\section{References}

1. Stein RA, Goetz RM, Ganea GM. Ceftobiprole: a new beta-lactam antibiotic. Int J Clin Pract 2009; 63: 930-943.

2. Pang Z, Raudonis R, Glick BR, Lin TJ, Cheng Z. Antibiotic resistance in Pseudomonas aeruginosa: mechanisms and alternative therapeutic strategies. Biotechnol Adv 2019; 37: $177-192$.

3. Zowawi HM. Antimicrobial resistance in Saudi Arabia. An urgent call for an immediate action. Saudi Med J 2016; 37: 935-940.

4. Holmes NE, Tong SY, Davis JS, van Hal SJ. Treatment of methicillin-resistant Staphylococcus aureus: vancomycin and beyond. Semin Respir Crit Care Med 2015; 36: 17-30.

5. Purrello SM, Garau J, Giamarellos E, Mazzei T, Pea F, Soriano A, et al. Methicillin-resistant Staphylococcus aureus infections: a review of the currently available treatment options. J Glob Antimicrob Resist 2016; 7: 178-186.

6. Bruniera FR, Ferreira FM, Saviolli LR, Bacci MR, Feder D, da Luz Gonçalves Pedreira M, et al. The use of vancomycin with its therapeutic and adverse effects: a review. Eur Rev Med Pharmacol Sci 2015; 19: 694-700.

7. Li B, Webster TJ. Bacteria antibiotic resistance: new challenges and opportunities for implant-associated orthopedic infections. J Orthop Res 2018; 36: 22-32.

8. Mpenge MA, MacGowan AP. Ceftaroline in the management of complicated skin and soft tissue infections and community acquired pneumonia. Ther Clin Risk Manag 2015; 11: 565-579.

9. Flamm RK, Duncan LR, Hamed KA, Smart JI, Mendes RE, Pfaller MA. Ceftobiprole activity against bacteria from skin and skin structure infections in the United States from 2016 through 2018. Antimicrob Agents Chemother 2020; 64: e02566-e02519.
10. Pfaller MA, Flamm RK, Mendes RE, Streit JM, Smart JI, Hamed KA, et al. Ceftobiprole activity against Gram-positive and -negative pathogens collected from the United States in 2006 and 2016. Antimicrob Agents Chemother 2018; 63: e01566-e01518.

11. Pfaller MA, Flamm RK, Duncan LR, Streit JM, Castanheira M, Sader HS. Antimicrobial activity of ceftobiprole and comparator agents when tested against contemporary Grampositive and -negative organisms collected from Europe (2015). Diagn Microbiol Infect Dis 2018; 91: 77-84.

12. Giacobbe DR, De Rosa FG, Del Bono V, Grossi PA, Pea F, Petrosillo N, et al. Ceftobiprole: drug evaluation and place in therapy. Expert Rev Anti Infect Ther 2019; 17: 689-698.

13. Hawser S, Kothari N, Karlowsky JA, Wiktorowicz T, Hamed K. In vitro susceptibility testing of ceftobiprole against 880 European respiratory tract infection isolates of methicillinresistant Staphylococcus aureus followed by whole genome sequencing of ceftobiprole-resistant isolates. Diagn Microbiol Infect Dis 2020; 96: 114978.

14. Falcó V, Burgos J, Almirante B. Ceftobiprole medocaril for the treatment of community-acquired pneumonia. Expert Opin Pharmacother 2018; 19: 1503-1509.

15. Antonelli A, Giani T, Coppi M, Di Pilato V, Arena F, Colavecchio OL, et al. Staphylococcus aureus from hospitalacquired pneumonia from an Italian nationwide survey: activity of ceftobiprole and other anti-staphylococcal agents, and molecular epidemiology of methicillin-resistant isolates. $J$ Antimicrob Chemother 2019; 74: 3453-3461.

16. Carnesecchi G, Lobello R, Liggieri L, Palmero C, Pescetto L, Morelli P, et al. In vitro activity of ceftaroline and ceftobiprole against methicillin-resistant Staphylococcus aureus with decreased susceptibility to vancomycin isolated in paediatric patients. $J$ Chemother 2018; 30: 338-341.

17. Zhanel GG, Lam A, Schweizer F, Thomson K, Walkty A, Rubinstein E, et al. Ceftobiprole: a review of a broad-spectrum and anti-MRSA cephalosporin. Am J Clin Dermatol 2008; 9: 245-254.

18. Bogdanovich T, Ednie LM, Shapiro S, Appelbaum PC. Antistaphylococcal activity of ceftobiprole, a new broadspectrum cephalosporin. Antimicrob Agents Chemother 2005; 49: 4210-4219.

19. David MZ, Dryden M, Gottlieb T, Tattevin P, Gould IM. Recently approved antibacterials for methicillin-resistant Staphylococcus aureus (MRSA) and other Gram-positive pathogens: the shock of the new. Int J Antimicrob Agents 2017; 50: 303-307.

20. Magiorakos AP, Srinivasan A, Carey RB, Carmeli Y, Falagas ME, Giske CG, et al. Multidrug-resistant, extensively drug-resistant and pandrug-resistant bacteria: an international expert proposal for interim standard definitions for acquired resistance. Clin Microbiol Infect 2012; 18: 268-281.

21. CLSI. Performance standards for antimicrobial susceptibility testing (28th ed.) CLSI supplement M100. Clinical and Laboratory Standards Institute; [Updated 2018; 2020 July 19]. Available from: https://clsi.org/media/1930/m100ed28_ sample.pdf

22. EUCAST. Ceftobiprole: Rationale for the clinical breakpoints (version 1.0). [Updated 2016; 2018 May 16]. Available from: https://eucast.org/

23. Grau S. Safety and tolerability of ceftobiprole. Rev Esp Quimioter 2019; 32: 34-36. 
24. Mahmoud E, Al Mansour S, Bosaeed M, Alharbi A, Alsaedy A, Aljohani S, et al. Ceftobiprole for treatment of MRSA blood stream infection: a case series. Infect Drug Resist 2020; 13 : 2667-2672.

25. Morroni G, Brenciani A, Brescini L, Fioriti S, Simoni S, Pocognoli A, et al. High rate of ceftobiprole resistance among clinical Methicillin-resistant Staphylococcus aureus isolates from a hospital in Central Italy. Antimicrob Agents Chemother 2018; 62: e01663-e01618.

26. Farrell DJ, Flamm RK, Sader HS, Jones RN. Ceftobiprole activity against over 60,000 clinical bacterial pathogens isolated in Europe, Turkey, and Israel from 2005-2010. Antimicrob Agents Chemother 2014; 58: 3882-3888.
27. Horcajada JP, Montero M, Oliver A, Sorlí L, Luque S, GómezZorrilla $S$, et al. Epidemiology and treatment of multidrugresistant and extensively drug-resistant Pseudomonas aeruginosa infections. Clin Microbiol Rev 2019; 32: e00031-e00019.

28. Rossolini GM, Dryden MS, Kozlov RS, Quintana A, Flamm RK, Läuffer JM, et al. Comparative activity of ceftobiprole against Gram-positive and Gram-negative isolates from Europe and the Middle East: the CLASS study. J Antimicrob Chemother 2011; 66: 151-159.

29. Bilman F, Turhanoğlu M. Antimicrobial activity of ceftaroline and ceftobiprole tested against MRSA isolates from Turkey in 2016. IOSR-JPBS 2016; 11: 52-56. 